\title{
Recognition Persian Handwritten Characters using Hough Transform
}

\author{
Neda Aashrafi Khuzani ${ }^{\# 1}$, Maryam Mahmoodi ${ }^{\# 2}$ \\ ${ }^{1,2}$ Department of computer, Meymeh branch,Islamic Azad University, Meymeh, Iran
}

\begin{abstract}
Today, expansion of computers influences in the business world has forced organizations to think about using computers to process and manage large amounts of information. Artificial intelligence provides approaches and algorithms for image processing. Using this algorithm, the computer can have the ability to identify the various components of an image.

The present study presents a method for detecting Persian handwritten characters using Hough transform. One of the main characteristics of the Hough transform is to detect the lines, circles and other shapes that have analytic relationships. Using this method along with documents image processing, data fields written in paper documents can be separately extracted and then use them for storage, processing, management and reporting. For instance, it considers discrete Persian handwritten characters as objects in a scene, and then attempts to diagnoses them using the Hough transform as well as neural network. According to the performed tests and obtained results, the accuracy of $70 \%$ was yielded in detection of characters.
\end{abstract}

Keywords-image processing, neural network, edge detection, skeletonizing, Hough transform.

\section{INTRODUCTION}

At the present time, doing the most of the works using computers is highly noticed due to the time and cost savings benefits. Additionally, widespread penetration of computers into the trade world and increasing and multipurpose use of them has prompted organizations to think about using the computers to process and manage large amounts of information. One of the main problems in this context is to provide the images of documents, process their images and derivate information.

Artificial Intelligence provides methods and algorithms for Image Processing. By using these algorithms, the computer is able to recognize and identify the various components of an image. Another part of the artificial intelligence world is related to identification of the recognized components. One of the applications of these algorithms is to recognize characters as well as written numbers and texts in the image. This method is called Optical Character Recognition or Optical Character Reader or briefly OCR, designed to detect and recover the alphabet by means of the computer. Detection of Persian handwritten characters is one of the important issues discussed in the field of OCR. Reading the manuscripts, this system which is called Intelligent Character Recognition or briefly ICR is a more advanced mode of OCR which is capable of reading the handwritten texts in addition to reading the typographical texts.

Handwritten scripts are usually written in two discrete and continuous forms on paper documents. Continuous scripts are the same usual writing mode. Discrete scripts are similar to the registration forms, in which characters of a statement are written individually on alternate squares. Using this method along with documents image processing, data fields written in paper documents can be separately derived and then used for storing, processing, managing and reporting. The present study will propose a method for detection of Persian handwritten characters using Hough transform. Applying this approach accompanied by documents image processing, data fields written in paper documents can be separately derived.

The Hough transform is a new technique which can be used to analyze isolated features of a particular shape within an image. Hough transform is most commonly used for the detection of regular curves such as lines, circles, ellipses, etc. Actually, Hough transform can convert the Cartesian space of image into parametric space which defined based on analytical equations belonged to the geometric shapes in the space of image. According such transform, each point in $\mathrm{x}-\mathrm{y}$ space corresponds to a line in parameter space and corresponding line with the points which are placed on same line pass through one unique point in the parameter space.

Using this technique the points that many lines pass through them can be determined as lines and be introduced in $x-y$ space and also required parameters to detect lines can be achieved through parameter space. $[2,3,7]$

Therefore, we study the following steps.

1) Yielding an image

2) Preprocessing

3) Hough Transform

4) Analyzing the results obtained by Hough Transform using neural network

According to the processes, the scene analysis process through Hough transform is composed of two main steps including image processing and neural network. As a practical example, the Persian handwritten characters images are employed and then they can be diagnosed. To identify the allowed forms of a character, there is a basic need to prepare a database. Different types of characters are entered in the considered databases. 


\section{HOUGH TRANSFORM}

Hough Transform is an appropriate technique for images with high brightness and contrast; however it is found with decreased applicability for vertical shaded images. It transforms between Cartesian space and some parameter spaces, in which a straight line can be introduced. A line can be presented by the shortest distance from its origin ( $r)$ and direction (s).

$$
r=x \cos (\theta)+y \sin (\theta)
$$

This, however, means that a point in Cartesian space is corresponded with a Sine wave in $r-\theta$ where we search a point with the highest sinusoidal sharing. In many practical situations a unique intersection can hardly be determined; however a group of intersections can be diagnosed[2,3,7].

An object can be determined in this transform if the formula and geometric equation of the item is available (straight lines and conic sections). Among the characteristics of this transform are:

- It is strong against noise and lack of information.

- This transform is used in human eye, too.

- It can act well on noisy images with interference or failure.

It is usually applied to images with determined lines and edges and preferably binary images[1,2,3,4,7,9].

\section{DESCRIPTION OF METHODS AND TESTING}

At the beginning of the objects discovery in the scene, targeted digital images projection should be gained by which the location of each object is detected and then the image is extracted using different processing methods. The image processing techniques are utilized in order to perform the preprocessing operation. The operation of the scene processing begins with the preparation of digital image which here implemented on the images of handwritten characters, and then continued by the recognition of the character location in the image and finished by deleting the rest of the components in the image (so only the characters are remained). Meaningful objects of the image are recognized, and then the results of detection will be extracted and finally the analysis of obtained features will be performed.

Hough transform is employed to features extraction. Before using the Hough transform the main image should be firstly converted into a gray scale image, and then the binary image should be obtained. Subsequently, edges should be found on binary image and skeletonizing and thinning must be implemented on the image to obtain input Hough Transform. In order to perform mentioned steps we'll convert the required digital image to an image with 256 gray scales (Figure 1 illustrates respective digital image). Then, preprocessing operation is be done in order to achieve the most appropriate input for Hough transform in which image complement initially should be gained. Because we frequently prefer to have objects in white color in dark background meanwhile black color is considered as the absence of the object (Figure 2 depicts the supplement image). Use of proper filter is recommended to obtain binary image before the conversion of image into binary image. Following the implementation of the filter, we transform the gray image into the binary image. When the binary image is yielded, we may encounter multiple shapes within the image other than characters which are totally considered as noise. Accordingly, in the next step, the noises involved in image should be taken away as much as possible prior to beginning the analysis of image which mostly includes the borders around each characters etc $[1,4,5,6,9]$.

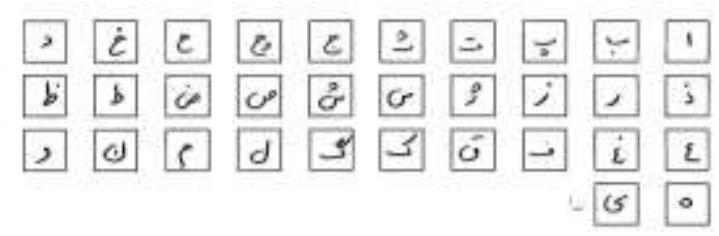

Figure 1 targeted digital image

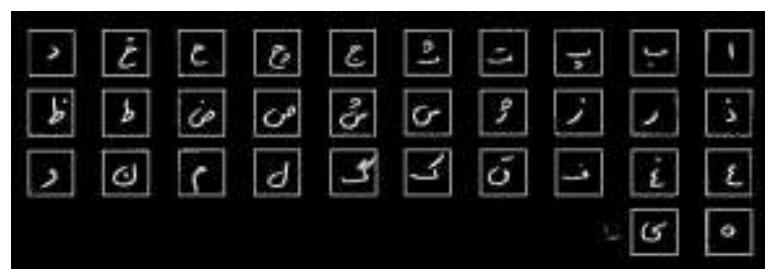

Figure 2 complement figure

Next, due to the fact that the analysis of whole characters is practically impossible and also is not suitable for applications, we deal with characters isolation and check each character as an individual object within image, i.e. we attribute an assigned label to each digit in the image. Thereby, the images related to each object (each character) in image (alphabetic characters) can be checked individually. One of the isolated characters has been represented in Figure 3.

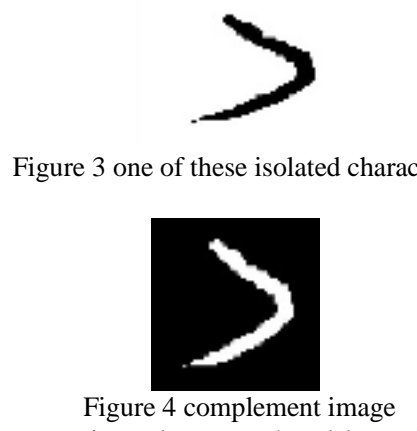

The rest of the mentioned steps should actually be carried on the characters. After the characters are yielded, the next step will be "preprocessing". The suitable input image for Hough transform is skeleton (thinned image) or image edge or spure image. 


\section{A. Detection of edge}

Edge is the boundary between regions with relatively distinct features of gray scale. Basic theory in most methods of the edge detection is to calculate a local derivative operator. The magnitude of the first derivative define pixel whether falls on the edge or not while the sign of the second derivative determine the pixel is located on the dark or light side of edge. The second derivative at the middle point of each edge encounters with a zero crossing which provides a strong path to determine the location of image edges. The magnitude of the first derivative at each point of the image is equal to the greatness of the two-dimensional gradient function. Second derivative can be measured using the Laplacian, too. In general, edge detection using Gradient operators act well when there is sharp change in the brightness of the image edges and the noise is relatively low (Figure 5) [5,6].

\section{B. Sceletonizing}

For smoothing or skeletonizing phase we use mathematical morphology. Mathematical morphology is a useful method for shapes detection. This method can break up complex shapes into meaningful parts or separate them from non-original parts. The basic binary morphological operators were used in order to recognize the lines in the image (Figure 6).

\section{C. spurring}

Spurring techniques are considered as the essential supplement for thinning or skeletonizing algorithms. Thinning algorithms leave the parasitic components in the image that should be removed through post-processing. This issue is done using spurring [1]. In the next section, we will employ obtained images as input of the Hough transform[5,6].

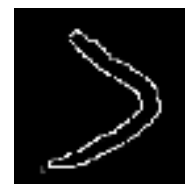

Figure 5 detected edge on image

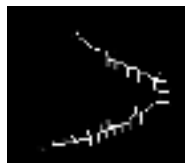

Figure 6 image skeleton

\section{HOUGH TRANSFORM AND FINDING LINES ON THE IMAGE}

A character in the yielded image contains a number of lines that lines detection can be performed through Hough transform. Figures 7 and 8 show the output of the Hough transform.

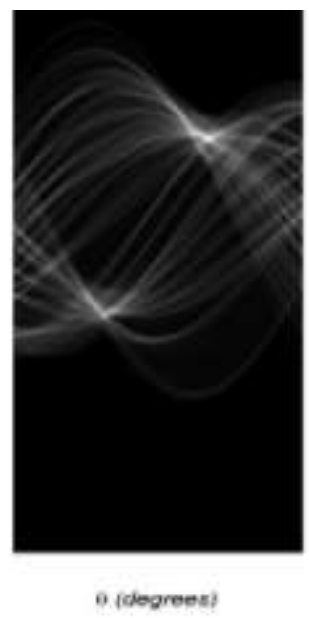

Figure 7 Hough transform of a skeletonized

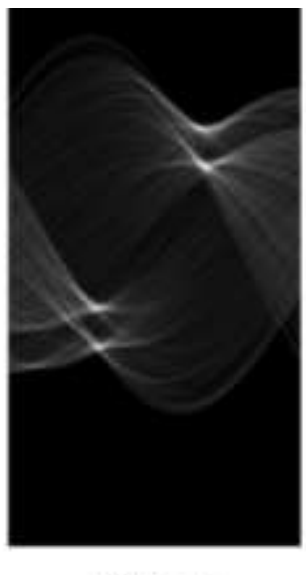

o (dingiensi)

Figure 8 Hough transform of edge detection

A. Finding location and characteristics of the lines by assessing the peaks

The first step is defined as peak detection when using the Hough transform for the purpose of line connection and detection. Try to finding an understandable set of distinct peaks in Hough cannot be serious, because of some smoother in digital image space, in addition to the fact that the edges are not perfectly straight in conventional images. Hough transform peaks may place in more than one cell. One strategy to solve this problem is explained as follows:

1. Find the Hough transform cell with the highest value and record its location.

2. Suppress (set to zero) Hough Transform cells in the immediate neighborhood of the maximum found in step 1.

3. Repeat until desired number of peaks has been found, or until a specified threshold has been reached. 
Figure 9 shows the peak position and four characteristics of the lines used in the analysis stage[5,6].

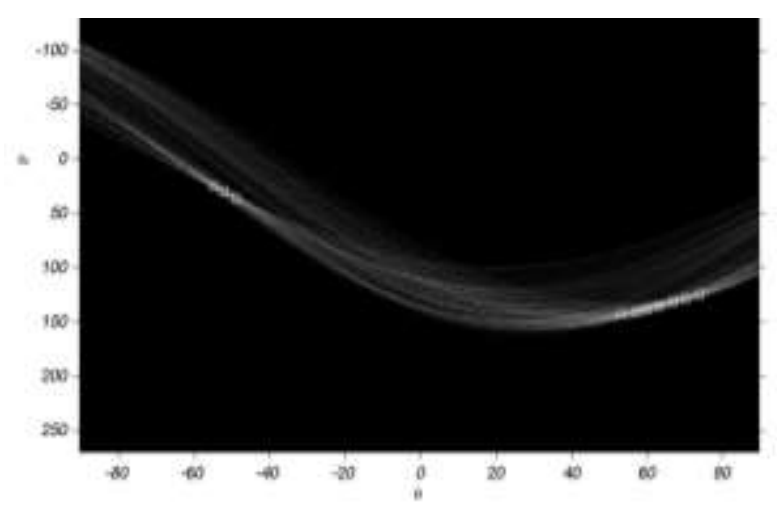

Figure 9 the position of peaks in skeletonizing mode

\section{B. Hough Transform Line Detection and Linking}

Firstly, a set of peaks has been identified in the Hough Transform, it remains to be established if there are line segments related with those peaks, where they start and end. For each peak, the first step is to discover the location of all nonzero pixels in the image that contributed to that peak.

\section{FEATURES ANALYSIS USING NEURAL NETWORKS}

In this section, the first step is to discover the most relevant databases. The database is based on the records induced by lines characteristic for The 32 alphabet "A" to "y" belonged to the different scripts. It is worth mentioning that the higher number of scripts may contribute to the further accuracy of learning activity and also testing the neural network. Database in accordance with its basic definition is explained as an interrelated united set of data interacting with each other under the specific rules. Dataset is introduced as a constituent of the database. Here, the dataset contains tables of characters records. The fields of these tables contain the lines features and each record is associated with a particular character[8].

First, MLP neural network is performed using the dataset. MLP network is designed in a training with 20 and 32 neurons, respectively in the input and output layer. The operation begins with choosing several neurons in the middle layer and then continues by an appropriate number of learning cycles and also selecting the features of alternative scripts of characters (as a train set) and one extra handwriting (as a test set).

One of the gained results is presented in Figure 10. The number of three different databases containing characteristics of detected line is utilized based on the Hough transform. These properties may vary with the edge detection preprocess, skeletonizing or spure having the duty of taking extra pixels away. In general, the accuracy of implemented network when dealing with data has been estimated about $70 \%$ in the best test.

Graph indicates the ideal characters in comparison to the signs obtained by neural network where the recognition system and targeted characters are shown with $\mathrm{O}$ and + symbols, respectively. Figure 10 describes data gained through implementing network using pre-processing method by creating skeleton.

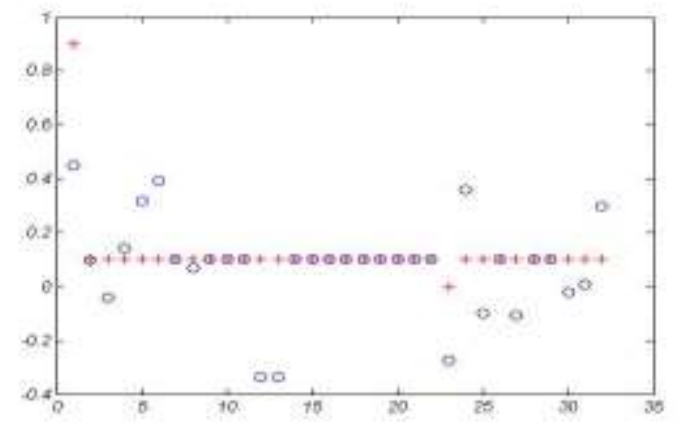

Figure 10 pre-processing method through skeletonizing

According to the results of implementation, the best accuracy is obtained when the spurring method (spure) were used as pre-processing.

\section{CONCLUSION}

As seen earlier, the lines of the objects within the scene were favorably detected using the Hough transform. Then we test the data set of MLP neural network through lines peaks detection and placing the characteristics of each line within the image and then we reached the accuracy of $70 \%$. Indeed, we investigated the Recognition of Persian handwritten characters and got the desired result. Taking into account the methods used as preprocessing, the spurring techniques lead to better results.

\section{REFERENCES}

[1] A.Amin, "Off-line arabic character recognition: the state of the art", Pattern Recognition, Volume. 31, No .5, p. 517-530, 1998

[2] D.H.Ballard., "Generalizing the Hough Transform to detect arabitrary shapes", Pattern Recognition, Volume. 13, No .2, pp. 111-122, 1981.

[3] S.R.Deans., "Hough Transform from the Radon Transform', PAMI(3), No. 2, pp. 185-188, March 1981.

[4] M.Fakir., C.Sodeyama, " On the recognition of arabic scripts using features selected in the Hough transform space", Advanced session of the IIEEJ on Doc. Proc. And Digital Imaging, pp. 231-234, 1992.

[5] ] R.C.Gonzalez., R.E.Woods., S.L.Eddins, ” Digital Image Processing by Using Matlab", 2004.

[6] R.C.Gonzalez., R.E.Woods., S.L.Eddins, ” Digital Image Processing ”, 2005.

[7] J.Illingworth., J.V.Kittler., " A Survey of the Hough Transform”, CVGIP(44), No. 1, pp. 87-116, October 1988.

[8] D.klerfors., “ Artificial Neural Networks", school of Business \& Administration, Saint Louia Univercity, 1998

[9] S.touj., N.essoukri ben amara., H.amiri., “ generalized hough transform for arabic obtical character recognition", Proceedings of the seventh international conference on documet analysis and recognition, (ICDAR 2003) 0-7695-1960-1/03\$17.00@ 2003 IEEE. 